\title{
Histopathological Changes Associated with Cutaneous Leishmaniasis in Patients with Baghdad Boil/South of Iraq
}

\author{
Amal Khudair Khalaf \\ Dept. Microbiology | college of Medicine | University of Thi-Qar
}

\begin{abstract}
Cutaneous leishmaniasis is an endemic disease in Iraq and it is become epidemic in Nassirriyah city recently/ South of Iraq.

The following histopathological study were achieved in microbiology dept. at college of Medicine in University of Thiqar, targeting Nassiriyah city in south of Iraq where Baghdad boil is highly distributed during a period of time at end of April 2016. several of tissue (skin) biopsies were obtained by dermatologist under sterilizing condition and at Al-Hussain teaching hospital where the patients have entered for treatment . the result explain the following changing :
\end{abstract}

- marked epidermal hyperplasia with hyperkeratosis .

- two small granulomas of macrophages some with vaculation in the dermis .

- sever dermal hyperplasia and foliculitis also hyperkeratosis and scab formation.

- marked hyperplasia of epidermis, hyperkeratosis and scab formation .

Key words : coetaneous leishmaniasis, Baghdad boil, histopathology

\section{Introduction}

The parasitic disease of Leishmaniasis caused by haemoflagellate Leishmania tropica. It is widespread and may cause serious health problems in communities throughout the Mediterranean regions and the Middle East, including Iraq ${ }^{[1-3]}$. There are an estimated 12 million cases worldwide, and there are about 1.5 million new cases of cutaneous leishmaniasis each year, of which over 90\% occur in Afghanistan, Algeria, Iran, Iraq, Saudi Arabia, Syria, Brazil and Peru ${ }^{[4]}$. Old World disease primarily is caused by Leishmania tropica minor in urban areas and Leishmania tropica major in dry desert areas ${ }^{[3]}$.

In Iraq, two species are present: $L$ tropica, the agent of anthroponotic cutaneous leishmaniasis (ACL), and

\section{Corresponding author:}

Amal Khudair Khalaf

Mobile: (07802521845)

Email: amalkhudair111@yahoo.com
L. major, the agent of zoonotic cutaneous leishmaniasis (ZCL). Both ACL and ZCL were reported as causative agents of leishmaniasis in Iraq, but ACL is found mainly in suburban areas ${ }^{[5]}$. The disease is epidemiologically unstable, with large and unpredictable fluctuations in the number of cases. The total incidence rate of cutaneous leishmaniasis in Iraq varies from 2.3 / 100000 to 45.5 / $100000^{[5]}$. Cutaneous leishmaniasis is characterized by one or more cutaneous lesions on areas where sandflies have fed. Persons who have cutaneous leishmaniasis have one or more sores on their skin. The sores can change in size and appearance over time. They often end up looking somewhat like a volcano, with a raised edge and central crater. A scab covers some sores. The sores can be painless or painful. Some people have swollen glands near the sores (for example, in the armpit if the sores are on the arm or hand). ${ }^{[6]}$

\section{Materials and Method}

- Skin biopsy : Biopsies were taken from patients by the dermatologist and placed in $10 \%$ of formalin to prepare it for tissue processing. ${ }^{(9)}$ 
- Histology : Tissues biopsy were processed according to ${ }^{(9)}$, briefly the tissue from the skin collected and placed in $10 \%$ formalin for histopathological studies and dehydrated by several dilutions of ethanol alcohol dealcoholization with xylol, then embedded with paraffin wax blocked (3-5) mm thickness sections were obtained by microtome. The sections were put on glass slides deparaffinised with xylol, rehydrated by alcohol and stained by hematoxyline and eosin.

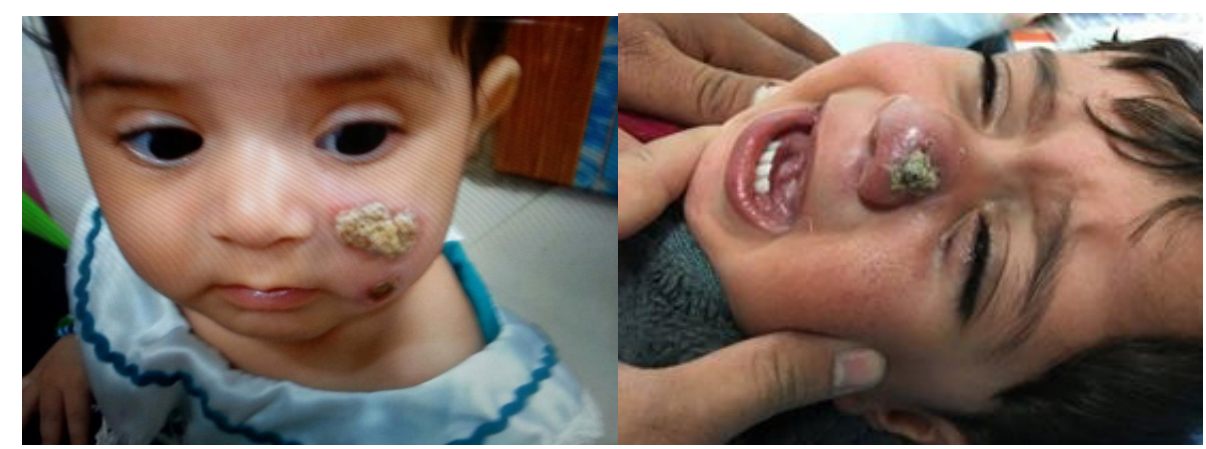

Fig(1) : explain the presence of Baghdad boil or cutaneous leishmaniasis

\section{Results}

The following histopathological changing has associated with Baghdad boil, the clinical infection with cutaneous leishmaniasis caused by the protozoan Leishmania tropica, as illustrated in the following figures;


Fig (2) : marked epidermal hyperplasia with hyperkeratosis (10X)


Fig(3): small granuloma in the dermis consist of macrophages and fibroblast at the periphery (40X) 

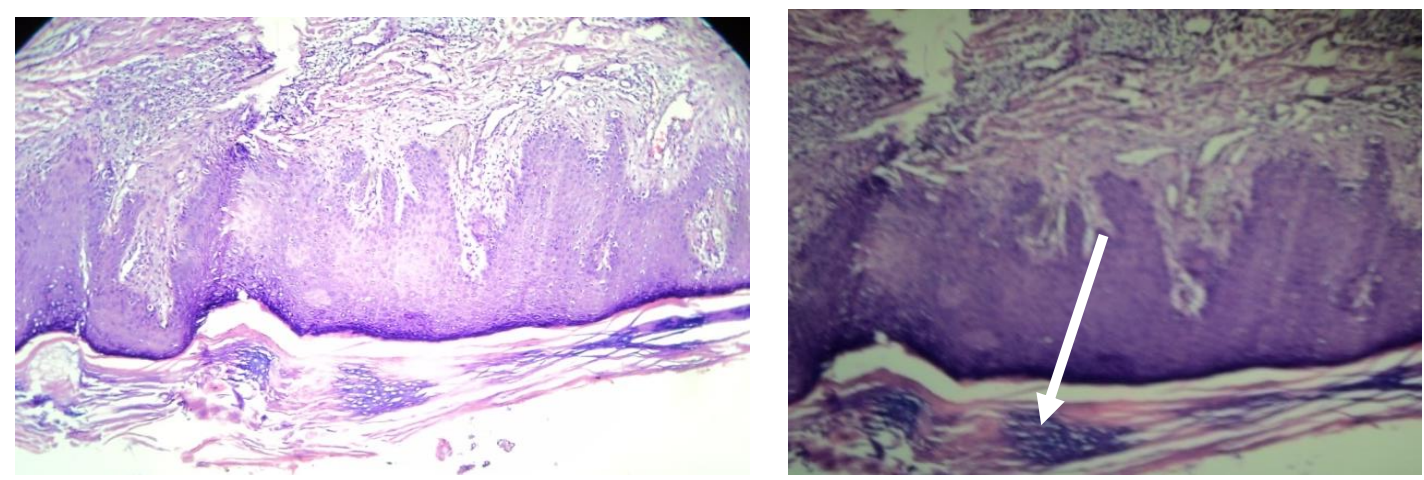

Fig (4) marked hyperplasia of epidermis, hyperkeratosis and scab formation (10X)
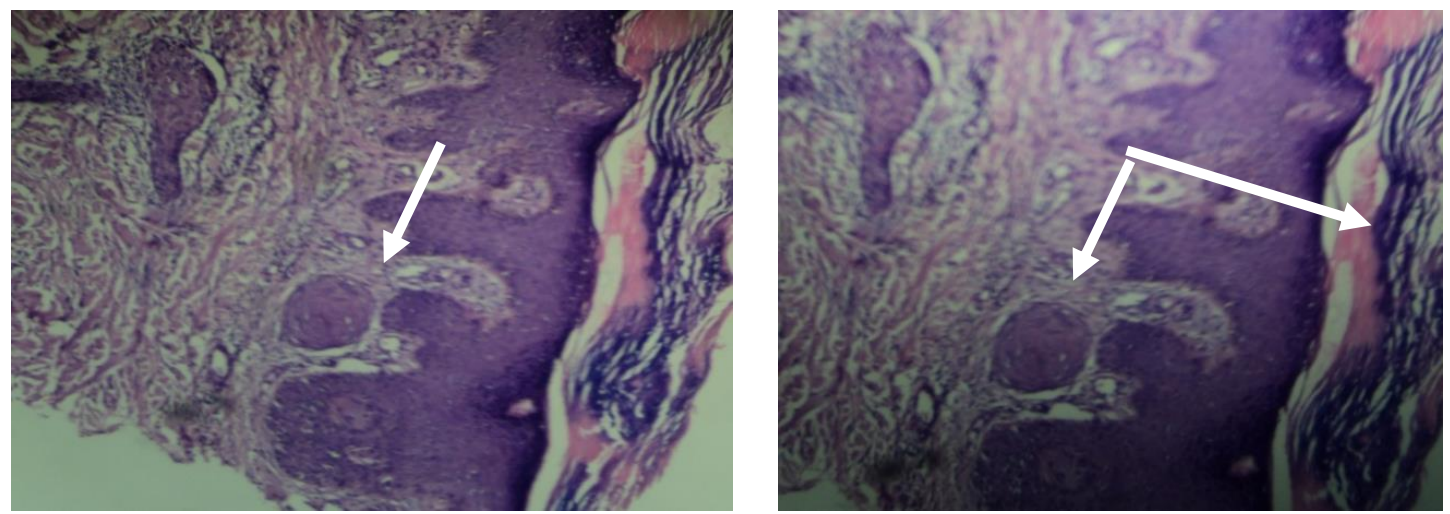

Fig (5) : marked hyperplasia of epidermis, hyperkeratosis and scab formation, keratin nest in the dermis (10X)
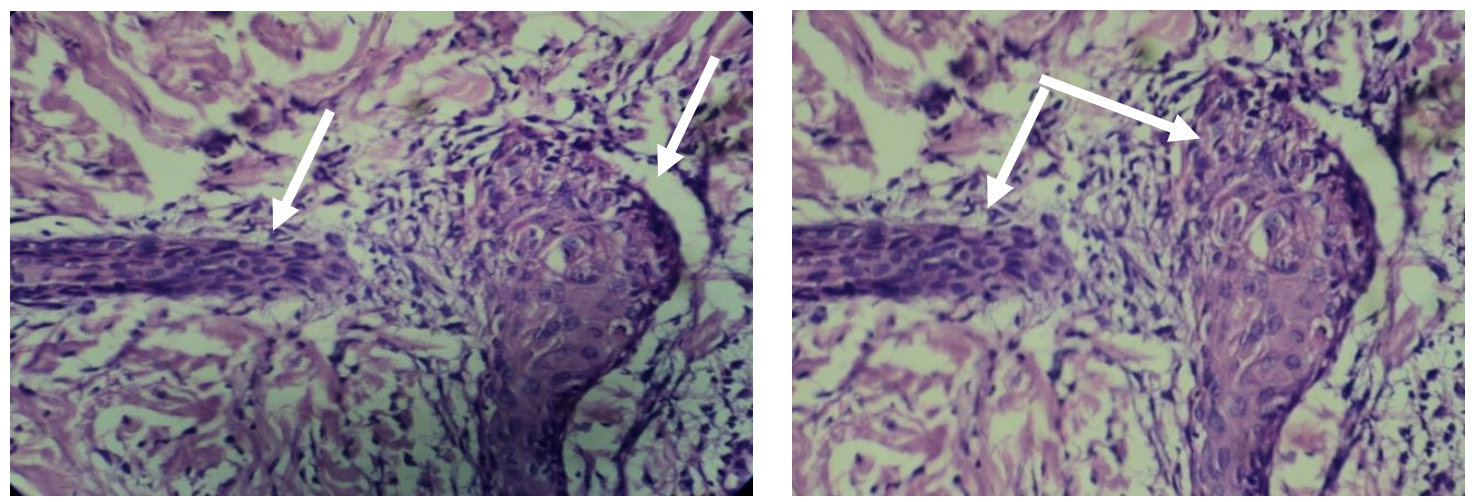

Fig (6): two small granulomas of macrophages some with vaculation in the dermis (40X)

\section{Discussion}

The skin covers the human body and is constantly exposed to the environment. As a barrier against infection, the skin not only forms a physical hindrance but also serves as a very important immunological organ. cutaneous leishmaniasis appears in variable patterns clinico-pathologically, and sometimes a simple erythemic lesion, shows (LD) bodies and in few cases has been diagnosed clinically malignant and remained untreated for years. The CL clinically presents according 
to the stage of infection and the clinical type of disease basically an ulcer appearing at the site which heals slowly, takes months to years depends upon the host immunity.

Cutaneous leishmaniasis, a wide clinical and histological spectrum. The histological picture in CL differs according to the stage of infection and the clinical type and host immunity. There is evolution of the lesion as it progresses from a papule and nodule into a soft boggy, crusted plaque or nodule ${ }^{(12)}$. The lesion breaks up after 3-4 months into a well circumscribed ulcer, which heals slowly over a period of 3 to 12 months. The histological spectrum in CL has wide range with a great variation in morphology. The appearances may range from predominance of leishmanial granuloma with macrophages showing epithelioid. Granuloma formation results from macrophages activation into epithelioid cells, considering clinicopathological relationship (11). The histological picture in CL differs according to the stage of infection and the clinical type. A consistent finding is a moderate to heavy dermal infiltrate of lymphocytes, plasma cells, and macrophages. In approximately $80 \%$ of cases, epithelioid cell granulomas with giant cells and a rim of lymphocytes are present. It is important to search for amastigotes, which are diagnostic (13,14). Histopathological changing of skin during cutanous leishmaniasis explain the granulomatous reaction in the site of infection together with presence of amastigote stage or LD bodies in the lesion based on the current study. The essential feature of CL pathology is the colonization by amastigotes of cells of the mononuclear phagocytic system and the resulting granulomatous inflammatory response. A granuloma is defined as a compact collection of mature mononuclear phagocytes, not necessarily accompanied by accessory features such as necrosis. Conceptually granuloma evolves in three stages: 1) the development of an infiltrate of young mononuclear phagocytes; 2) the maturation and aggregation of these cells into an unorganized granuloma; and 3 ) the potential maturation of these cells into an epithelioid or organized granuloma ${ }^{(7-10)}$

Cutaneous Leishmaniasis presents a spectrum of manifestations both clinically and histologically. Lesions can present as nodule, plaque or ulcer mostly present on exposed sites ${ }^{(8,9)}$. Histopathological findings in acute CL include dermal infiltrate predominantly consisting of macrophages containing large number of leishmania organism called LD bodies ${ }^{(5,6)}$ In addition plasma cells and dense mixed inflammatory cell infiltrate are also present in dermis. The morphology of LD bodies in histopathological sections were rounded with a nucleus and kinetoplast, in some sections spindle shape form similar to smear morphology were detected ${ }^{(7,8)}$.

Ethical Clearance: The Research Ethical Committee at scientific research by ethical approval of both environmental and health and higher education and scientific research ministries in Iraq

Conflict of Interest: The authors declare that they have no conflict of interest.

Funding: Self-funding

\section{References}

1. CDC. Update CL in US. Military personnel. Southwest / Central Asia, 2002-2004. MMWR .2004;53:264-265.

2. Hepburn ,NC ;Cutaneous leishmaniasis: an overview. JPGM .2003;49:50-54.

3. Markle, WH Makhoul, $\mathrm{K}$.Cutaneous leishmaniasis Recognition and Treatment. American Family Physician. 2004;69: 1-9.

4. Ashford RW, Desjeux P, de Raadt,P . Estimation of population at risk of infection and number of cases of leishmaniasis. Parasitol Today.1992; 8:104-105.

5. WHO .Communicable Disease Working Group on Emergencies, HQ Division of Communicable Disease Control, EMRO, WHO OFFICE, Baghdad.2003.

6. Arfan B, Rahman ,S .Correlation of clinical, histopathological, and microbiological finding in 60 cases of cutaneous leishmaniasis. IJDVL .2006;72:28-32.

7. Akilov OE, Khachemoune A, Hasan T. Clinical manifestations and classification of Old World cutaneous leishmaniasis. Int J Dermatol .2007; 46(2): 132-42.

8. Gazozai SU, Iqbal J, Bukhari I, Bashir S. Comparison of diagnostic methods in cutaneous leishmaniasis (histopathology compared to skin smears).Pak J Pharm Sci 2010; 23(4):363-6.

9. Rahman SB, Bari A U. Histopathological patterns of Cutaneous Leishmaniasis with clinical correlation seen in Pakistan. PakArmed Forces Med J .2003; 53(2):142-7.

10. Mashhood AA, Khan I, Nazir S. Histopathological spectrum of cutaneous leishmaniasis in North 
West Frontier Province. J Pak Assoc Derma. 2004; 14(4):210-4.

1. Gazazali ,S., Iqbal J., Bukhari ., B. Comparison of diagnostic methods in cutaneous leishmaniasis . Pak. J. Pharm. Sci.,.2010;4, 363-366.

11. Khawer S Bushra and Altaf, S .Histological grading patterns in patients of cutaneous Leisiamaniasis. JCPSP, 2007;17: 650-653.
12. Rahman SB, Bari AU, Khan AA. A new focus of cutaneous leishmaniasis in Pakistan. J Pakistan Assoc Dermatol .2003;13:3-6.

13. Mashhood AA, Khan IM, Nasir S, Agha H, Saleem $\mathrm{M}$, Imran A. Fine needle aspiration cytology versus histopathology in the diagnosis of cutaneous leishmaniasis in Pakistan. J Coll Physicians Surg Pak 2005; 15:71-3. 